\title{
Investigation of Fluidborne Noise Reduction in Automotive Hydraulic Power Steering Systems
}

\author{
Chuan-Chiang Chen ${ }^{1 *}$, Olakunle Harrison ${ }^{2}$ and Adrian K. McKinney ${ }^{3}$ \\ ${ }^{1}$ Mechanical Engineering Department, California State Polytechnic University Pomona, USA \\ ${ }^{2}$ Mechanical Engineering Department, Tuskegee, AL 36088, USA
}

${ }^{3}$ Engineer Proctor \& Gamble Company, Cincinnati, OH 45242, USA

\begin{abstract}
The performance of a pulse suppressor, currently used to alleviate pump-induced noise in automotive power steering systems, was characterized in this research. A hydraulic test system was developed to evaluate the noise attenuation effects of a pulse suppressor as well as other devices. Four pressure transducers were used to measure the dynamic pressures in the pressure line at different locations (two before and two after the suppression device); thus it was possible to determine the transfer matrix elements relating line pressures to noise. The transmission loss (the criteria used to determine the noise isolation performance of a noise reduction device) for this device was also estimated. The test system was verified by using a 0.52-meter section of steel tubing. Good agreement was obtained between the experimental and theoretical matrix elements.

The results show that the direction of a pulse suppressor plays a role in the attenuation of the pressure wave. Therefore, the symmetry of the suppressor cannot be assumed. Compared to other noise reduction devices (reinforced rubber hose, coaxial tuning cable and hose) the pulse suppressor provides good transmission loss. However, the overall system response is a function of frequency. It is suggested that various noise reduction devices be combined to achieve the greatest noise reduction.

The methodology can be used to optimally reduce the pump-induced noise and vibration in hydraulic power steering systems during the design stage. With the experimentally determined transfer matrix for each device, the relationship between two ports can readily be obtained by matrix multiplication. The analysis presented in this investigation can be also applied to other fluid power systems (i.e., engine cooling systems and HVAC systems).
\end{abstract}

Keywords: Fluidborne noise; Pulse suppressor; Tuning cable; Transfer matrix; Transmission loss

\section{Introduction}

Automobile consumers are now linking the NVH (Noise, Vibration and Harshness) characteristics of an automobile to its quality. In it attempts to improve vehicle quality and increase revenues; the automotive industry has expended considerable efforts in minimizing or reducing noise and vibration from all sources. In general, the main source of noise and vibration in the automobile is the engine. Over the years, automotive engineers have made considerable gains in reducing engine noise. As a result of the dominant engine noise being alleviated, other sources of noise become more apparent. One of those sources is the hydraulic power steering, which emits a low-pitched humming noise that is most prominent when the car is stationary, the engine is running, and the steering wheel is fully turned.

In previous studies [1,2] showed that power steering noise correlates with the fluid flow ripples generated by the power steering pump. To reduce the pump-induced noise in the power steering system, several approaches were used in the automotive industry; for example, the addition of a section of hose, a restrictor, or coaxial tuning cable with hose. Among these devices, the coaxial tuning cable with hose is the most common device used to reduce the amplitude of pressure waves based on the principle of destructive interference $[3,4]$. Since the attenuation mechanism of the tuning cable and coaxial hose is not fully understood and verified, experimental analysis of the attenuation effects of the tuning cable $[5,6]$ were performed using four pressure transducer measurements to experimentally determine the transmission loss. The results show that inclusion of the tuning cable and coaxial hose in the tubing system provides high attenuation over the frequency range of interest. More recently, a pulse suppressor was added to alleviate the fluidborne noise in the hydraulic system. It functions as an accumulator to dampen out the fluid pulsations. In research presented by [7] a suppressor is depicted making major reductions to the pressure signal generated by the pump in the time domain. However, the frequency characteristics of the suppressor were not investigated.

In this paper, a test scheme based on the two-load method was used to determine the transmission loss in order to assess the performance of different noise reduction device used in power steering transmission line. By measuring the pressure signals before and after the test section, the transfer matrix relating the pressure and flow velocity were also estimated. Since the overall response of the complete automotive power steering system is dependent on the acoustic characteristics of all components as well as the device's placement in the transmission linen, the transfer matrices can be used to incorporate other system components to further predict the complete system response.

\section{Methodology}

The approach is based on the two-load method $[8,6]$ to determine

${ }^{*}$ Corresponding author: Chuan-Chiang Chen, Associate Professor, Mechanical Engineering Department, California State Polytechnic University Pomona, USA E-mail: chuanchen@csupomona.edu

Received June 04, 2012; Accepted June 23, 2012; Published June 25, 2012

Citation: Chen CC, Harrison O, McKinney AK (2012) Investigation of Fluidborne Noise Reduction in Automotive Hydraulic Power Steering Systems. J Appl Mech Eng 1:110. doi:10.4172/2168-9873.1000110

Copyright: ( 2012 Chen CC, et al. This is an open-access article distributed under the terms of the Creative Commons Attribution License, which permits unrestricted use, distribution, and reproduction in any medium, provided the original author and source are credited. 


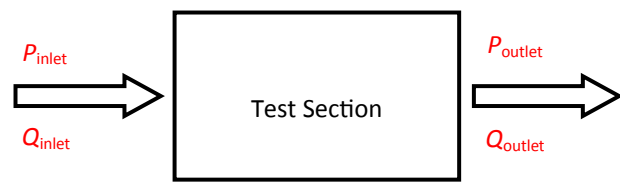

Figure 1: Representation of a two-port model.

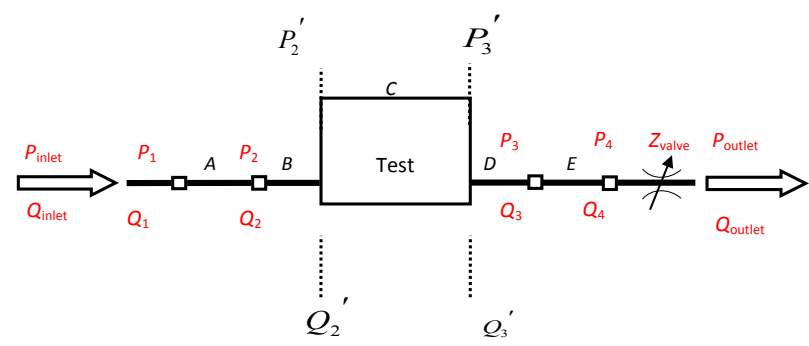

Figure 2: A two-port model illustrating the implementation of four pressure transducers used to determine the transfer matrix elements, where sections $A, B, D$, and $E$ represent the reference tubes used to find the unknown elements of the test section $\mathrm{C}$. Using a loading valve, the boundary condition 1 is obtained, where Zvalve represents the termination impedance of the valve.

the transfer matrix and estimate the transmission loss of hydraulic noise reduction devices. The test section with two anchored ends then can be represented by a two-port model shown in (Figure 1) where $P$ and $Q$ are the pressure and volumetric flow rate at the inlet and outlet of the test section.

The two-port model can then be described by the following transfer matrix $[T]$ relating the pressure $(P)$ and volumetric flow velocity $(Q)$ upstream to those downstream.

$$
\left[\begin{array}{l}
P \\
Q
\end{array}\right]_{\text {outlet }}=\left[\begin{array}{ll}
T_{11} & T_{12} \\
T_{21} & T_{22}
\end{array}\right]_{\text {section }}\left[\begin{array}{l}
P \\
Q
\end{array}\right]_{\text {inlet }}
$$

Since the test section represents an unknown hydraulic noise reduction device, four pressure transducers were used to determine the transfer matrix elements of the test section. Therefore, a modified fur port model is given in Figure 2.

Now, using the transfer matrix to evaluate section $A$ of the twoport model the matrix becomes

$$
\left[\begin{array}{l}
P_{2} \\
Q_{2}
\end{array}\right]=\left[\begin{array}{ll}
T_{11} & T_{12} \\
T_{21} & T_{22}
\end{array}\right]_{A}\left[\begin{array}{l}
P_{1} \\
Q_{1}
\end{array}\right]
$$

Through manipulation, $Q_{2}$ can be obtained in terms of $P_{1}$ and $P_{2}$

$$
Q_{2}=T_{21}^{A} P_{1}+T_{22}^{A}\left[\frac{P_{2}-T_{11}^{A} P_{1}}{T_{12}^{A}}\right]
$$

Applying the transfer matrix to section $B, P_{2}^{\prime}$ and $Q_{2}^{\prime}$ can be determined with the known $P_{2}$ and $Q_{2}$ :

$$
\left[\begin{array}{c}
P_{2}^{\prime} \\
Q_{2}^{\prime}
\end{array}\right]=\left[\begin{array}{ll}
T_{11} & T_{12} \\
T_{21} & T_{22}
\end{array}\right]_{B}\left[\begin{array}{l}
P_{2} \\
Q_{2}
\end{array}\right]
$$

Similarly, relating the $P$ and $Q$ at the two ports of section $D$, gives:

$$
\left[\begin{array}{l}
P_{3} \\
Q_{3}
\end{array}\right]=\left[\begin{array}{ll}
T_{11} & T_{12} \\
T_{21} & T_{22}
\end{array}\right]_{D}\left[\begin{array}{l}
P_{3}^{\prime} \\
Q_{3}^{\prime}
\end{array}\right]
$$

Multiplying the inverse matrix $[T]_{D}$, the unknown pressure and volumetric flow velocity of section $D$, produces:

$$
\left[\begin{array}{l}
P_{3}^{\prime} \\
Q_{3}^{\prime}
\end{array}\right]=\left[\begin{array}{ll}
T_{11} & T_{12} \\
T_{21} & T_{22}
\end{array}\right]_{D}^{-1}\left[\begin{array}{l}
P_{3} \\
Q_{3}
\end{array}\right]
$$

To find $Q_{3}$, the transfer matrix is applied to section $\boldsymbol{E}$ (similar to section $\boldsymbol{A}$ ) and $Q_{3}$ is:

$$
Q_{3}=\frac{P_{4}-T_{11}^{E} P_{3}}{T_{12}^{E}}
$$

Now, for the section $C$, the relationship between the two ends is:

$$
\left[\begin{array}{l}
P_{3}^{\prime} \\
Q_{3}^{\prime}
\end{array}\right]=\left[\begin{array}{ll}
T_{11} & T_{12} \\
T_{21} & T_{22}
\end{array}\right]_{C}\left[\begin{array}{c}
P_{2}^{\prime} \\
Q_{2}^{\prime}
\end{array}\right]
$$

To solve for the transfer matrix elements $\left(T_{11}, T_{12}, T_{21}\right.$, and $\left.T_{22}\right)$ with the known $P_{2}, Q_{2}, P_{3}, Q_{3}$ and two more equations are needed (i.e., 4 unknowns, 4 equations). Therefore, a second boundary condition is needed, to obtain another set of pressures and volumetric flow velocities for the section $\boldsymbol{C}$.

Similarly, applying the transfer matrix to sections $A, B, D$, and $E$, the unknown flow velocities and pressures were determined. Therefore, for boundary condition 2, the transfer matrix of section $\mathrm{C}$ is given by

$$
\left[\begin{array}{c}
P_{3}^{\prime \prime} \\
Q_{3}^{\prime \prime}
\end{array}\right]=\left[\begin{array}{ll}
T_{11} & T_{12} \\
T_{21} & T_{22}
\end{array}\right]_{C}\left[\begin{array}{l}
P_{2}^{\prime \prime} \\
Q_{2}^{\prime \prime}
\end{array}\right]
$$

Now, arranging the four linear equations (from equations 8 and 9) into matrix form gives:

$$
\left[\begin{array}{l}
P_{3}^{\prime} \\
Q_{3}^{\prime} \\
P_{3}^{\prime \prime} \\
Q_{3}^{\prime \prime}
\end{array}\right]=\left[\begin{array}{cccc}
P_{2}^{\prime} & Q_{2}^{\prime} & 0 & 0 \\
0 & 0 & P_{2}^{\prime} & Q_{2}^{\prime} \\
P_{2}^{\prime \prime} & Q_{2}^{\prime \prime} & 0 & 0 \\
0 & 0 & P_{2}^{\prime \prime} & Q_{2}^{\prime \prime}
\end{array}\right]\left[\begin{array}{l}
T_{11} \\
T_{12} \\
T_{21} \\
T_{22}
\end{array}\right]_{C}
$$

Once the transfer matrix elements are determined, the transmission loss $(T L)$ can be expressed in $\mathrm{dB}$ scale [9]:

$$
T L=20 \log _{10}\left|\frac{T_{11}^{C}-T_{12}^{C} / Z_{c}-T_{21}^{C} Z_{c}+T_{22}^{C}}{2\left(T_{11}^{C} T_{22}^{C}-T_{12}^{C} T_{21}^{C}\right)}\right|
$$

In Figure 2, two sections of steel tubing are used to connect each end of the test section (sections $\mathrm{AB}$ and DE). Since the elastic properties of steel tubing are well defined, the transfer matrix for each section is modeled as: 


$$
\left[\begin{array}{c}
P \\
Q
\end{array}\right]_{\text {outlet }}=\left[\begin{array}{cc}
\cosh (\Gamma L) & -Z_{c} \sinh (\Gamma L) \\
-\frac{1}{Z_{c}} \sinh (\Gamma L) & \cosh (\Gamma L)
\end{array}\right]\left[\begin{array}{l}
P \\
Q
\end{array}\right]_{\text {inlet }}
$$

where $\Gamma=s \Omega / c$ is the propagation constant; $s=i \omega$ is the Laplacian operator; $\omega$ is the rotational frequency; $c$ is the speed of sound; and $\Omega=\left[1-\frac{2}{j r_{i} \sqrt{j \omega / v}} \frac{J_{1}\left(j r_{i} \sqrt{j \omega / v}\right)}{J_{0}\left(j r_{i} \sqrt{j \omega / v}\right)}\right]^{-1 / 2}$ is the coefficient which accounts for the viscosity effect [4].

\section{Experimental Setup}

A 2-hp electric motor is used to drive a ten-vane power steering pump via a multi-ribbed serpentine belt, to simulate the actual power steering hydraulic transmission system. The pump uses the Ford power steering fluid (Type F) and serves as the primary source of fluidborne noise in the circuit. Since the fluidborne noise is the main focus of this investigation, it was imperative to isolate the structural vibrations of the pump and motor from the test section. Therefore, as shown in figure 3 , the pump and motor were mounted on a separate concrete base from the test section. The bases were made to be as heavy as possible, to alleviate any excessive vibrations in the test system. Also, rubber padding was placed under both bases to dampen out any unwanted movements.

The pump speed is monitored via a photoelectric switch and reflector in conjunction with a dynamic signal analyzer. A pressure gauge is used to measure the static pressure in the circuit, to ensure differences in the boundary conditions.

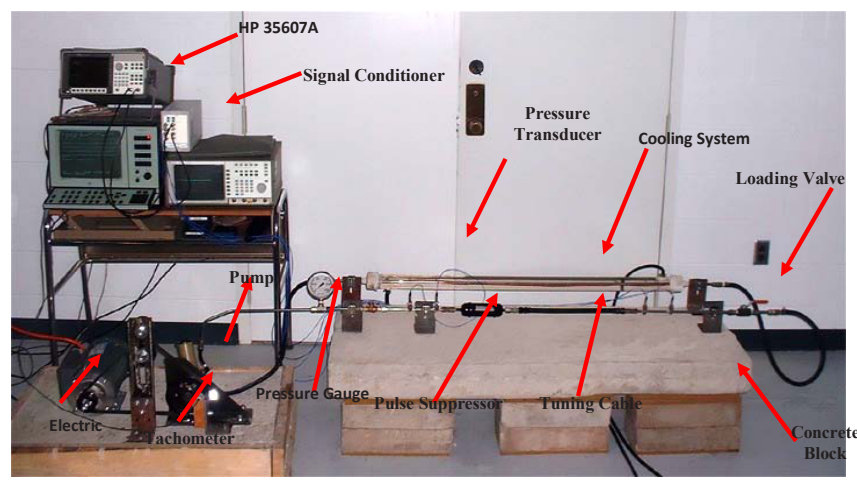

Figure 3: Experimental setup of a hydraulic test system.

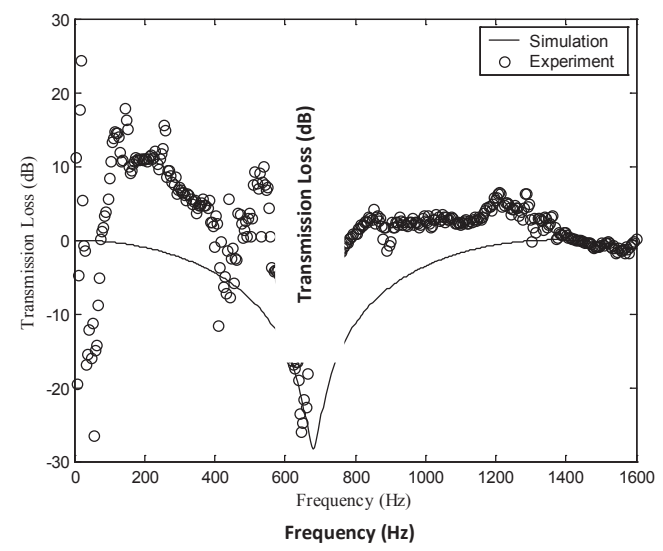

Figure 4: Transmission loss of a 0.52-meter-long section of steel tubing. upstream pipe and two on the downstream pipe connected to the test section, to measure the dynamic pressures (at measurement locations $P_{1}, P_{2}, P_{3}$, and $\left.P_{4}\right)$ of the power steering fluid. The distances between the measurement locations $P_{1}$ and $P_{2}, P_{3}$ and $P_{4}$ were determined to be 0.1 meters which is about $17 \%$ of the wavelength while taking the maximum frequency of interest to be $2 \mathrm{kHz}$ and the sonic speed of the fluid contained within the steel tubing to be about $1350 \mathrm{~m} / \mathrm{s}$.

To provide two boundary conditions a ball valve was installed, to serve as the loading valve (termination condition) in the circuit. The loading valve is used to increase and decrease the load being applied to the pump. While the pump is running; the change in load results in the two boundary conditions obtained: $\mathrm{BC} 1$ and $\mathrm{BC} 2$.

As a result of the circulation flow, excessive heat is produced in the hydraulic circuit because of the restricted flow and the increase in fluid friction of power steering fluid. A water-cooling system was built to dissipate the heat.

\section{Results}

A steel tubing system was used, to check the validity of the test system. Because the mechanical properties of steel are well defined, the test system was verified by comparing the theoretical analysis of Hastings and Chen (1993) to the experimental results obtained using the test system. Figure 4 is a comparison of the theoretical and experimental transmission loss for a 0.52-meter-long section of steel tubing. The experimental and theoretical data have a similar trend; this shows that the test system can be used to predict the transmission loss. The figure displays the minimum transmission loss at about $675 \mathrm{~Hz}$, which can be estimated from the numerical values of sonic speed and tubing length. These disturbances are a result of the flow discontinuities and the termination impedance of the system, which leads to high reflective waves. Also, the fluid properties were not readily obtained; some errors might contribute to the discrepancies.

The pulse suppressor was tested in the forward and reverse direction. Figure 5 shows the transmission loss of the suppressor in both forward and reversed directions. In this figure the pulse suppressor in the reversed direction has a higher transmission loss, than the pulse suppressor in the forward direction at higher frequencies.

The coaxial tuning cable and hose combination was also tested in the forward and reversed directions. With the transfer matrix determined, Figure 6 shows the comparison of the transmission loss

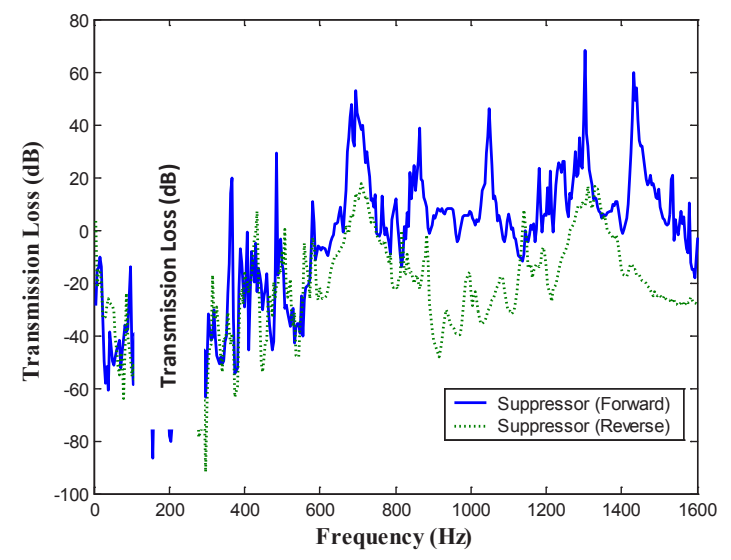

Figure 5: Comparison of transmission losses between two pulse suppressors. 


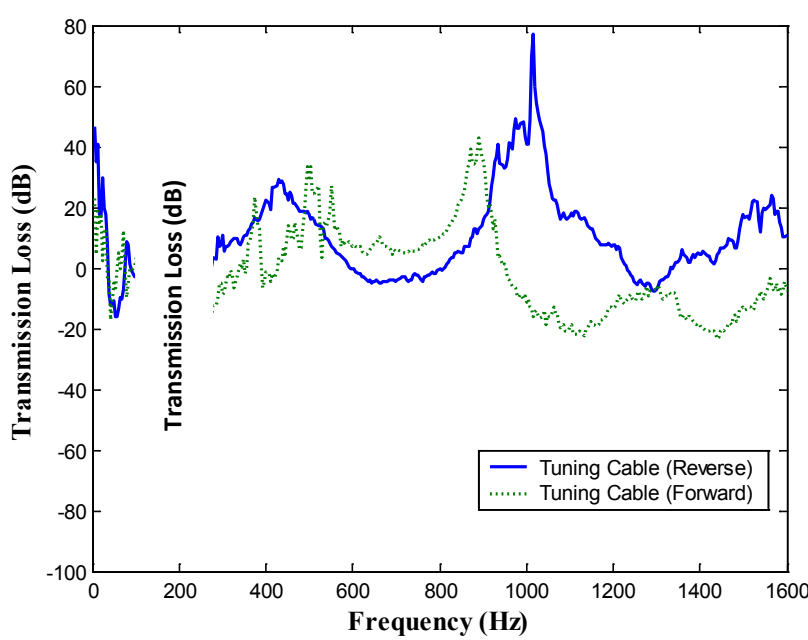

Figure 6: Transmission loss for the reverse and forward coaxial tuning cable and hose.

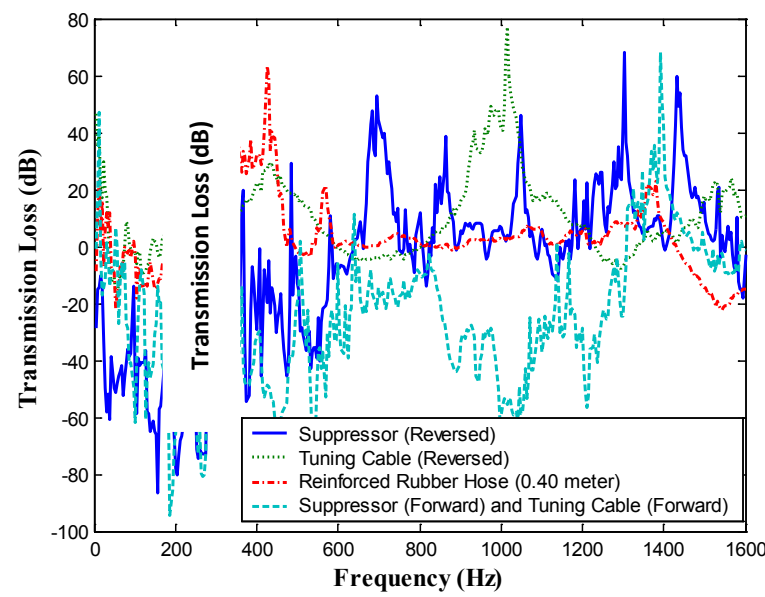

Figure 7: Comparison of the transmission loss of the main attenuation devices.

of the tuning cable in the forward and reversed directions. Overall the tuning cable in the reverse direction has a higher transmission loss resulting in a better attenuation of fluid-borne noise. However, at low frequencies there is only about a $10 \mathrm{~dB}$ difference in the transmission loss of the tuning cable in the forward and reversed directions.

Figure 7 compares the transmission loss of the main devices used in automotive systems: the pulse suppressor in the reversed direction, the tuning cable in the reversed direction, the 0.40 meter reinforced rubber hose, and the integrated suppressor - tuning cable. It can be seen that the overall transmission loss is a function of frequency.

Another parameter used to analyze the noise reduction performance is the pressure ratio the output pressure to the input pressure- $\mathrm{P} 4 / \mathrm{P} 1$, this ratio is highly dependent on the end condition. Figure 8 gives the comparison of the amplitude ratio of the above devices. Figure 8 displays that the integrated pulse suppressor and tuning cable has the lowest amplitude ratio, which results in better attenuation of fluid-borne noise under a certain termination condition. Therefore, an optimal design is needed for integrated devices in a system, which might achieve the best reduction of fluid-borne noise.

\section{Conclusions}

A hydraulic test scheme was developed to determine the transfer matrix elements (not shown here) and transmission loss of hydraulic noise reduction devices. The system was verified with theoretical analysis of a 0.52 meter long steel tube. The results showed fairly good agreement for the section of steel tubing and thus verified the feasibility of the testing system.

From the results of this study, it was found that the direction of a sound suppressor plays a role in the attenuation of the pressure wave. It was also shown that the symmetry of the suppressor cannot be assumed.

The tuning cable and hose system does have a higher attenuation than a hose without a tuning cable. Also, the direction of placing the tuning cable in the hose affects the overall response. It is concluded that the reversed tuning cable with hose can dampen out more fluid-borne noise, than the forward one.

The transmission loss of an integrated system including the pulse suppressor and tuning cable was determined. The system was evaluated with the suppressor and tuning cable both in the forward and reversed direction. The results show that further investigation is needed to optimize the attenuation performance of the integrated system.

The erratic data points in the graphs might result from the experimental errors (for example, the signal-to-noise ratio is not large enough to distinguish the signal from the noise disturbances.). This is because of the discontinuities existing in the hydraulic circuit which resulted in higher-amplitudes of reflected pressures and thus standing waves. Also, the ball valve termination, with a geometric which abruptly changes the flow direction, might have caused turbulent flow.

Overall, the transmission loss varies with the frequency (i.e., it is a function of frequency) for all different devices. An optimization of the system layout is needed to minimize the fluid-borne noise (i.e., change the combinations of the tuning cable, hose, and suppressor). With the availability of this study; however, the transfer matrices can be readily obtained via experiments. Optimal design can be achieved by computer simulation with the experimentally estimated transfer matrix data. The methodology can be also applied to other hydraulic power systems.

\section{Acknowledgements}

This work was funded by The Ford Motor Company.

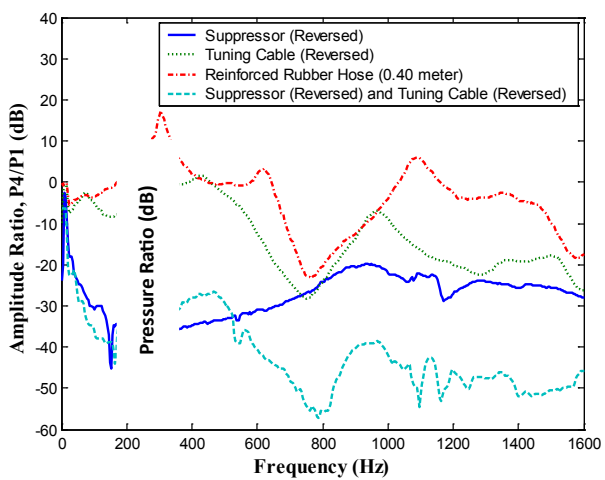

Figure 8: Comparison of the amplitude ratio of the main attenuation devices 
Citation: Chen CC, Harrison O, McKinney AK (2012) Investigation of Fluidborne Noise Reduction in Automotive Hydraulic Power Steering Systems. J Appl Mech Eng 1:110. doi:10.4172/2168-9873.1000110

Page 5 of 5

\section{References}

1. Smith J, Ferries G, Arbanas R (1995) An Analytical Control System Approach to Steering Shudder. SAE Paper, 951254.

2. Sciortino G, Bamdad-Soofi J (1997) Identification and Elimination of Steering Systems Squawk Noise. SAE Technical Paper 972058.

3. Klees G (1967) Attenuation Device. United States Patent.

4. Chen CC, Hastings M (1992) Noise reduction in Power Steering Transmission Lines. InterNoise Proc 121: 67-72.

5. Yu J, Popescu S. (2001) Measurements of Transmission and Attenuation Characteristics of Fluidborne Noise in Fluid Hoses with/ without Tuning Cables. SAE Technical Paper 2001-01-1610.
6. Chen C-C (2001) An Investigation of Noise and Vibration in an Automotive Power Steering System.

7. Wilkes R (1995) Reduction of Noise in Hydraulic Systems. SAE Technical Paper 952154

8. Yu J, Johnson F, Iwami F, Verrecchia N, Kojima E (1999) Experimental Evaluation for Fluidborne Noise Attenuation in Tuning Cables and Hoses of Automotive Power Steering Hydraulic Systems. SAE Technical Paper 199901-1777.

9. Munjal ML (1987) Acoustics of Ducts and Mufflers With Application to Exhaust and Ventilation System Design. Wiley-Interscience Publisher. 